\title{
A Bayesian approach to improving estimate to complete
}

\author{
Franco Caron $^{\mathrm{a}, *}$, Fabrizio Ruggeri ${ }^{\mathrm{b}}$, Beatrice Pierini ${ }^{\mathrm{a}}$ \\ "Department of Management, Economics and Industrial Engineering, Politecnico di Milano, Via Lambruschini 4/b, 20156 Milano, Italy \\ b CNR IMATI, Via Bassini 15, 1-20133 Milano, Italy
}

Received 26 November 2015; received in revised form 6 September 2016; accepted 15 September 2016

Available online 1 October 2016

\begin{abstract}
The capability to develop a reliable 'Estimate at Completion' from the earliest stage of project execution is essential in order to develop a proactive project management. In order to accomplish this aim, a model to formulate estimates at completion is presented which integrates through a Bayesian approach three knowledge sources: experts' opinions, data from past projects and the current performance of the ongoing project. The model has been applied to three Oil and Gas projects in order to forecast their final duration and cost. These projects are characterized by a high level of size, uncertainty and complexity representing a challenging test for the model. The results obtained show a higher forecasting accuracy of the Bayesian model compared to the traditional Earned Value Management (EVM) methodology.

O 2016 Elsevier Ltd. APM and IPMA. All rights reserved.
\end{abstract}

Keywords: Project control; Forecasting; Estimate to complete; Bayesian approach; Eamed value management; Oil \& Gas industry 


\title{
A Bayesian approach to improving Estimate to Complete
}

\begin{abstract}
The capability to develop a reliable 'Estimate at Completion' from the earliest stage of project execution is essential in order to develop a proactive project management. This paper provides a methodology to support the development of the Estimate at Completion in large engineering projects. In order to accomplish this aim, a model to formulate estimates at completion is presented which integrates through a Bayesian approach three knowledge sources: experts' opinions, data from past projects and the current performance of the ongoing project. The model has been applied to three Oil and Gas projects in order to forecast their final duration and cost. These projects are characterized by a high level of size, uncertainty and complexity representing a challenging test for the model. The results obtained show a higher forecasting accuracy of the Bayesian model compared to the traditional Earned Value Management (EVM) methodology. Moreover, the estimates at completion calculated using the Bayesian model are not point estimates such as those calculated by EVM. In fact, the Bayesian approach leads to a probability density function for the forecasted final cost and duration. Hence, the project manager obtains an indication of the degree of confidence about the expected value forecasted which results in better quality information available for the decision making process.
\end{abstract}

Keywords: project control, forecasting, estimate to complete, Bayesian approach, Earned Value Management, Oil\&Gas industry

\section{Introduction}


Forecasting is a critical process in project management since, relying upon sound estimates to complete, the project manager can steer the ongoing project in order to meet specific time and cost objectives (Dvir and Lechler, 2004). Without good forecasting there can be no rationale in making a decision and we will have to rely on project adaptability to emerging circumstances.

Planning and forecasting are strictly intertwined both in the early stage of the project life cycle when the project baseline must be determined and then throughout the entire life cycle when effective decisions must be made (Hogarth and Makridakis, 1981). In an Earned Value Management context, the role of the Estimate to Complete (ETC), i.e. how much money and time is needed in order to complete the project, is critical, since the information drawn from the ETC, is essential in order to identify suitable corrective actions in order to achieve the project objectives. This approach to project control corresponds to a feed-forward control loop (Anbari, 2003; Christensen, 1996), since analysis of the future informs present-day decisions.

At a given time in the project cycle, i.e. the time now (TN), a certain amount of the work has been completed (Work Completed, WC), while the rest of the work is to complete, corresponding to the Work Remaining (WR). The cost and time performance related to the Work Completed is known, while a forecast has to be developed for the WR (see fig.1).

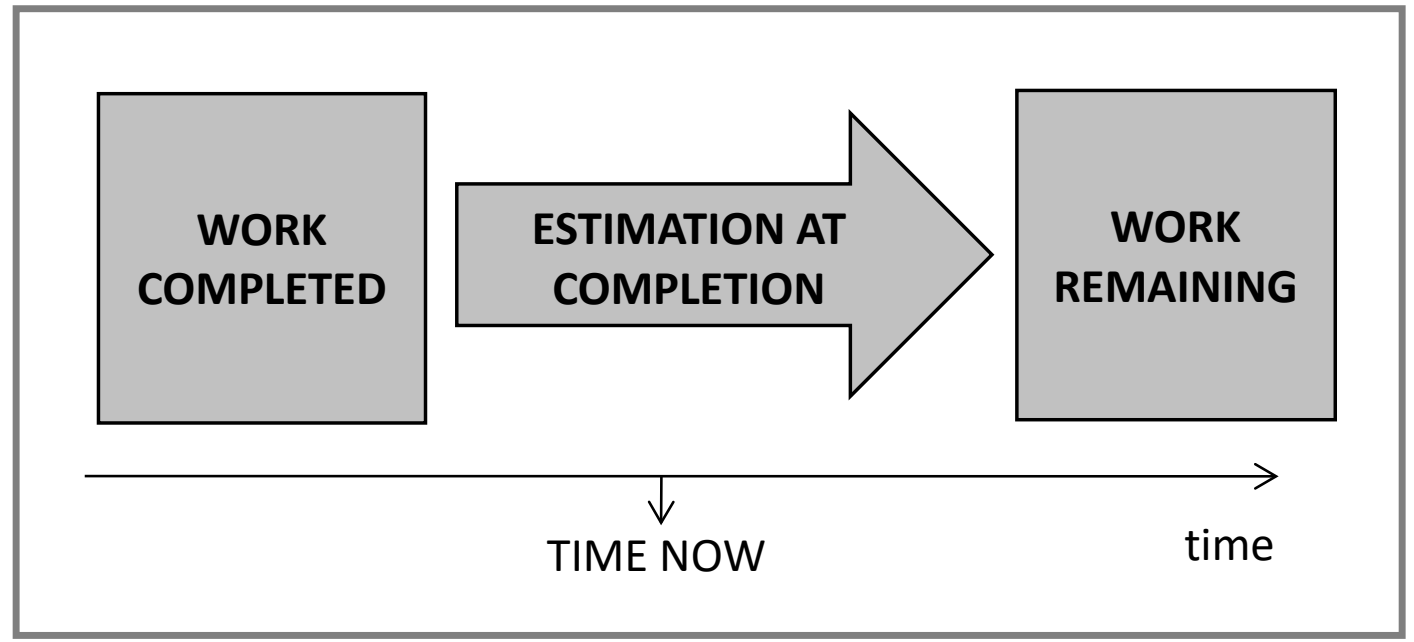

Figure 1 - Estimation at Completion at Time Now (internal view)

It should be noted that both the accuracy of the forecast of WR and the impact of the corrective actions that may be implemented based on the forecast will depend on the progress of the project 
at the Time Now. The effectiveness of the corrective actions is greater in the early stages of the project execution and progressively diminishes as progress increases: in fact, as progress increases, the degrees of freedom available to steer the project tend to reduce progressively. On the other hand, the capability to forecast the project final duration and cost follows an opposite trend. In fact, at an early stage in the execution phase, the knowledge available to the decision maker is scant and rapidly evolving; therefore, the capability to provide a reliable forecast is reduced, particularly if the forecast is only based upon the analysis of the performance of the ongoing project until the Time Now, without considering any other knowledge source.

From a survey analyzing the data of more than 300 mega-projects (Merrow, 2011), it appeared that in $201065 \%$ of the industrial projects with a minimum budget of 1 billion US dollars did not succeed in meeting the objectives of cost, duration and quality. Even though project management systems based on EVM have been extensively used in the recent years, failure to meet planned objectives is common, in particular in large engineering and construction projects such as in the oil \& gas industry (Merrow, 2011). However, it remains an open question whether these failures are due to a lack of project efficiency during the execution stage or to a lack of forecasting accuracy during the initial planning and control process. In the former case, both positive and negative deviations from the baseline should be expected, depending on the specific performance of the project. On the contrary, a systematic overrun in terms of cost and time may be easier explained as a weakness in the forecasting process since the beginning of the project. In the latter case, as a consequence, the methodologies commonly applied for forecasting purposes become the crux of the problem.

By using a set of case studies from the Oil \& Gas industry, this paper develops a Bayesian model to determine the estimate to complete for a project. The paper aims at multiple purposes:

- to utilize all the available knowledge in order to improve the forecasting process;

- to use a Bayesian approach in order to integrate diverse knowledge sources in a formal and rigorous way;

- to develop a Bayesian model capable of using data records related to WC, experts' judgments related to WC and data records related to similar projects completed in the past;

- to test the model in a set of case studies related to large and complex projects in the oil\&gas industry. 
The section two identifies the different knowledge sources available to feed the forecasting process and addresses how to mitigate any systematic bias during the process. The section three introduces the different approaches available to foresee the future performance of the project and in particular the use of the Earned Value Management system, which is a very popular technique frequently applied to determine the estimate at completion for a project, both in terms of cost and time. The section four introduces the general structure of a Bayesian model as a technique to integrate the knowledge contribution given by each knowledge source. The subsequent section five develops in detail the single components of the model: prior distribution, distribution based on experts' opinions, distribution based on past similar projects, definition of the weights given to the previous prior distributions when combining them, shape of the prior distribution, likelihood function, posterior distribution. Eventually, some results are given about the application of the model to some very complex and very risky projects in the oil \& gas industry. At the end, some conclusions are drawn to highlight the contribution given by the paper to the project control problem.

\section{Knowledge sources}

As mentioned above, all the available knowledge should be used in order to improve the planning and control process for a complex project (Caron, 2014; Reich et al., 2014; Schindler and Eppler, 2003).

In general, the knowledge available to the project team may be classified in two ways: explicit/ tacit and internal/external. Explicit internal knowledge corresponds to data records related to the work completed WC, allowing, as in the traditional EVM, for an evaluation of the performance trend at Time Now. Tacit internal knowledge is related to the experts' judgment about possible events/situations affecting the project's work remaining WR. Explicit external knowledge corresponds to data records about similar projects completed in the past. Tacit external knowledge concerns the identification of similarities between the current project and some past projects in order to transfer past data to the current project.

Depending on the types of knowledge used, three alternative approaches may be envisioned: 
- utilizing only data records related to $\mathrm{WC}$, by extrapolating the current performance trend into the future (i.e. the traditional EVM approach);

- adjusting the trend resulting from data records through experts' judgment about the expected performance during WR (Caron et al., 2013);

- integrating the internal view of the project, i.e. data records related to WC and experts' judgment related to WR, with data records deriving from similar projects completed in the past (this is the goal of this paper).

In the last approach, besides the use of internal knowledge, both explicit and tacit, external knowledge related to similar projects completed in the past can also be used (Lovallo et al., 2012). For instance, taking into consideration past experience should mitigate a possible "optimistic" bias in estimating future project performance (Lovallo and Kahneman, 2003). Firstly, Kahneman's and Tversky's studies (1979) show that a major source of forecasting failure, which influences the accuracy of final cost and duration estimates, is linked to an exclusively "internal" view approach, i.e. based only on knowledge deriving from inside the ongoing project. Then, the focus moves to the psychological and political factors introducing a bias in the planning process (Lovallo \& Kahneman, 2003).

Even more so, the need emerges to exploit all the available knowledge during the planning and control process, in order to minimize any bias. In fact, as shown in figure 1, the traditional control process often focuses only on data related to the ongoing project, corresponding to an exclusively “internal" view (Flyvbjerg, 2006). Integration between the knowledge stemming from the "internal" view and the knowledge stemming from the "external" view is needed, the latter related to the projects completed in the past (see figure 2). 


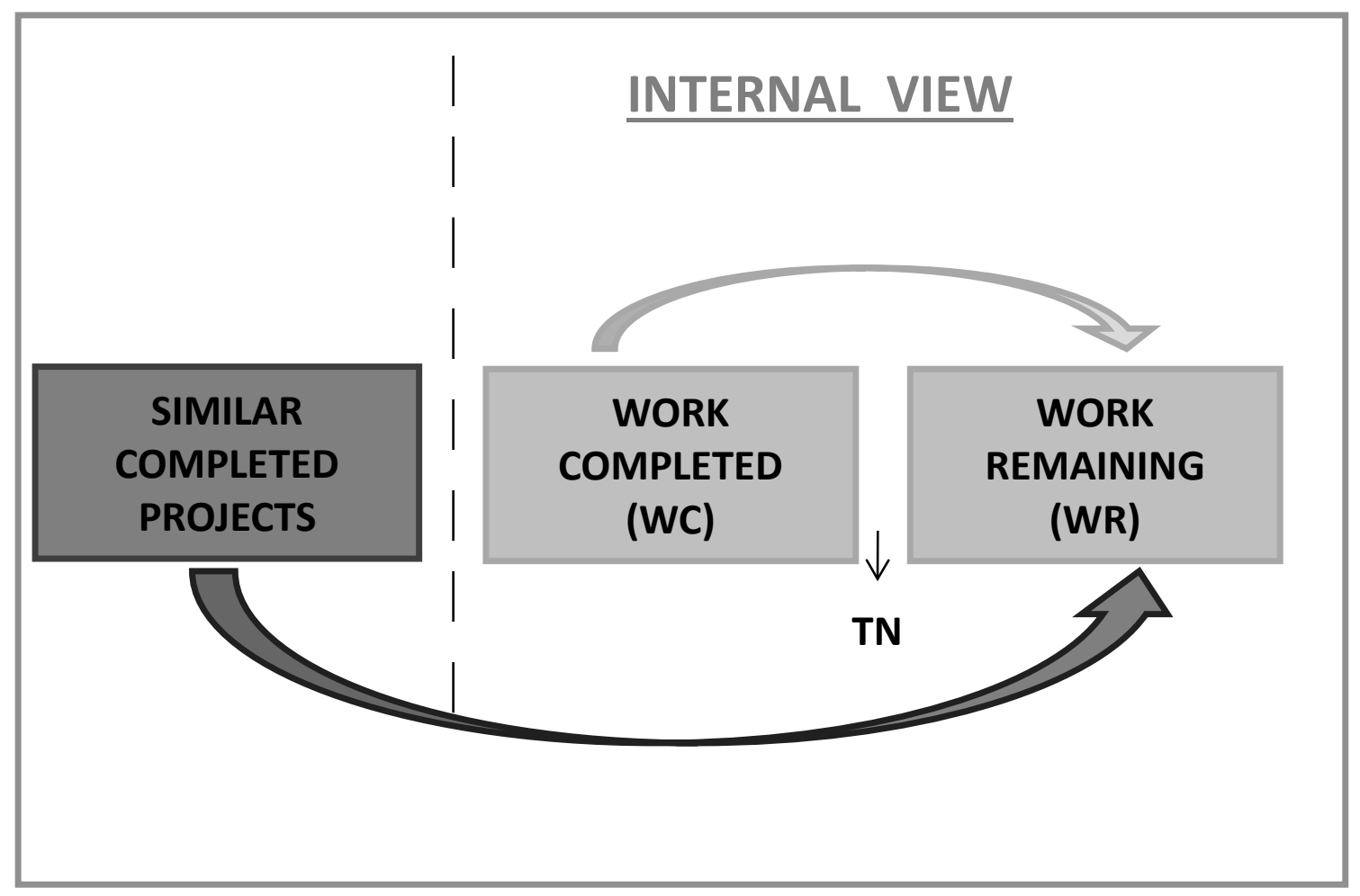

Figure 2 - Internal and external view

In fact, the current project can be seen as belonging to a cluster of similar projects developed by the company. Note that the selection of the cluster is basically subjective since it depends on the similarity criteria adopted (Savio \& Nikoloupolos, 2011; Green \& Armstrong, 2007). In fact, in some cases, similarity may indicate a strong ambiguity. For example, if a company has to estimate the cost of an investment in a new technology and, moreover, in an unfamiliar technological domain, should it take into account the set of highly innovative projects developed in different technological domains or the set of barely innovative projects but belonging to the same technological domain? Neither the former nor the latter option may be the best solution but both should be taken into consideration (Kahneman \& Tversky, 1979). Moreover, a similarity assessment should also consider the trade-off between using a large number of past projects, leading to the risk of including projects substantially different from the current one, and a small number of projects, leading to the risk of losing statistical significance. 


\section{Earned Value Management System}

The basis for any forecasting process is that the future has its seeds in the present (Kuosa, 2012). In a project, among the different approaches available for the forecasting process (Trend Analysis, Network Analysis, Pattern Analysis, Monte Carlo Simulation, Stakeholder Analysis, etc.) Trend Analysis is a very popular approach. The most popular version of the Trend Analysis applied to projects is the Earned Value Management (EVM). It is based on linear extrapolation since the performance related to WC is linearly extrapolated to WR (Fleming and Koppelman, 2006). The extrapolation normally concerns the parameters Cost Performance Index (CPI) and Schedule Performance Index (SPI). EVM is an efficient and often used performance measurement and reporting technique for estimating cost and time at completion (Project Management Institute, 2013; Marshall et al., 2008).

The following basic parameters are used in EVM, where TN indicates Time Now, i.e. the time along the project life cycle at which the control process is implemented:

- Planned Value (PV), the budgeted cost of work scheduled at TN;

- Earned Value (EV), the budgeted cost of work completed at TN;

- Actual Cost (AC), the actual cost of work completed at TN.

EVM was improved by Lipke $\left(2002_{a} ; 2000_{b} ; 2003\right)$, who introduced the concept of Earned Schedule (ES) for obtaining a measure of the schedule performance index based on time units and overcoming the flaws associated with a Schedule Performance Index SPI defined as the ratio between EV and $\mathrm{PV}$, both of them expressed in monetary terms. Earned Schedule is the time at which the EV value achieved at TN should have been obtained according to the project baseline. The new Schedule Performance Index $\mathrm{SPI}(\mathrm{t})$ at $\mathrm{TN}$, defined as the ratio between $\mathrm{ES}$ and $\mathrm{TN}$, represents a more effective approach, since it avoids the problem of convergence of the EV and PV values toward BAC (Budget at Completion) and consequently the unreliability of the estimate when the project progress increases (Lipke, 2006a; Lipke, 2006b).

The above three parameters and the ES, all of them evaluated at TN, allow for the calculation of a set of indices and variances at TN. The most important of these are: 
- Cost Performance Index

$\mathrm{CPI}=\mathrm{EV} / \mathrm{AC}$

- Cost Variance

$C V=E V-A C$

- Schedule Performance Index

$\mathrm{SPI}_{(\mathrm{t})}=\mathrm{ES} / \mathrm{TN}$;

- Schedule Variance

$S V_{(t)}=E S-T N$

Variances CV and SV(t) summarize the project's past performance during WC, while indexes CPI and $\mathrm{SPI}(\mathrm{t})$ may be used in order to extrapolate the current trend and estimate the future performance during WR (Anbari, 2003).

Many formulas for Estimate at Completion have been proposed during almost 50 years of applying EVM but none of them has proved to be always more accurate than any other one (Christensen, 1993). In the basic approach, the estimate of final cost (i.e. EAC) and final duration (i.e. TAC, Time at Completion) are based on the following equations:

$E A C=A C+(B A C-E V) / C P I_{f}$

where:

$\mathrm{AC}=$ Actual Cost at TN

BAC $=$ Budget at Completion

$\mathrm{EV}=$ Earned Value at TN

$\mathrm{CPI}_{\mathrm{f}}=$ Cost Performance Index estimated for the work remaining (WR)

$\mathrm{TAC}=\mathrm{TN}+(\mathrm{SAC}-\mathrm{ES}) / \mathrm{SPI}_{\mathrm{f}}$

where:

$\mathrm{TN}=$ Time Now

SAC $=$ Scheduled at Completion, i.e. the planned duration of the project

ES = Earned Schedule at Time Now

$\mathrm{SPI}_{\mathrm{f}}=$ Schedule Performance Index estimated for the work remaining (WR) 
It should be noted that future performance may significantly differ from past performance. The new performance indices $\mathrm{CPI}_{\mathrm{f}}$ and $\mathrm{SPI}(\mathrm{t})_{\mathrm{f}}$ have been introduced in equations 1 and 2 with reference to the Work Remaining and may consider a different evolution of the project performance. In fact, these indexes can take into account not only data records related to WC but also subjective expectations made by the decision makers about the WR. While the generic indices $\mathrm{CPI}$ and $\mathrm{SPI}(\mathrm{t})$ are related to the overall $\mathrm{WC}, \mathrm{CPI}_{\mathrm{f}}$ and $\mathrm{SPI}_{(\mathrm{t}) \mathrm{f}}$ are related to the overall $\mathrm{WR}$. In fact, relying only on past performance while developing a forecast could be misleading, since considering only past values of $\mathrm{CPI}$ and $\mathrm{SPI}_{(\mathrm{t})}$ is similar to driving a car whilst looking just in the rear view mirror, so making it impossible to dodge the obstacles that may lie on the road ahead. Hence the forward looking contribution given by experts' judgments reveals fundamental. Both equations 1 and 2 indicate that the values assigned to the performance indexes $\mathrm{CPI}_{\mathrm{f}}$ and $\mathrm{SPI}_{\mathrm{f}}$ play a critical role in obtaining an accurate estimate of the final cost and duration. In summary, forecasting capability can be improved by utilizing all the available knowledge about the performance indexes CPIf and SPIf (Liu \& Zu, 2007; Goodwin, 2005).

\section{Knowledge Integration - Bayesian Approach}

The Bayes Theorem represents a formal tool for the integration of explicit and tacit knowledge. In a Bayesian framework, the experts' preliminary opinions are an example of the use of subjective probability, which is the only probability applicable to non-repetitive processes such as projects. Subjective probability is defined as the degree of belief in the occurrence of an event, by a given person at a given time and with a given set of information. It should be noted that increasing the level of knowledge available may modify the value of probability assigned to a future event (De Finetti, 1937; Caron and al., 2013). Whilst the metaphor of 'frequency based' probability is throwing dice (i.e. a repetitive process), the metaphor of subjective probability may be the gamble (i.e. a unique process). In general, we can assume that any proactive action involving the future is a gamble, such as a corrective action implemented during project control. In fact, De Finetti defined probability as a price in a lottery. For instance, let's consider a lottery where, if the event E occurs, the better wins 1, if it doesn't happen the better wins 0 . How much is the better prepared to pay for accepting the 
lottery? That price is the subjective probability value associated to the event $E$ and the probability value corresponds to the degree of belief about event E occurring.

Several Bayesian models have been proposed in literature to formulate reliable forecasts (Palomo et al., 2006; Gardoni et al., 2007; Kim and Reinschmidt, 2009; Kim, 2015). In particular, the Bayesian adaptive model introduced by Gardoni et al. and the model by Kim and Reinschmidt aim at forecasting the actual duration of a project, by deriving the $S$ curve describing the progress of the ongoing project. In the first paper, the results show the importance of integrating the information extracted from a cluster of similar projects to obtain reliable predictions, when the project is in its early stage. In the second paper, the proposed method provides reliable time estimates since it is able to extract more information from the common monthly reports. Both methods provide a higher accuracy than the traditional EVM approach; however, both approaches focus uniquely on time performance and require quite complex computations, if compared to the extremely simple EVM formulas. A third paper (Kim, 2015) presents a new probabilistic project cost forecasting that systematically integrates project cost risk assessment and actual performance data in a project into a computationally efficient probabilistic cost forecast.

The essence of Bayesian inference is the use of probability to describe our state of knowledge about some event or parameter of interest (e.g. the cost of an item). A prior distribution (based on expert's tacit knowledge) is updated by means of experimental observations (data records) collected during the execution process, in order to obtain a posterior distribution, integrating both knowledge types. For instance, the project team may assume a prior distribution of the final budget overrun, based on subjective expectations about the development of the current project, and this prior distribution may be updated based on the actual performance of the current project until Time Now (Caron et al., 2013).

The above statement is easier to understand if the formulation of the Bayes theorem is considered. The Bayes theorem can be stated as:

$f\left(\mu \mid y_{1}, y_{2}, \ldots, y_{n}\right) \propto L\left(\mu ; y_{1}, y_{2}, \ldots, y_{n}\right) \cdot f(\mu)$ 
where we omit the normalizing constant (i.e. probability integrates to one) in order to enlighten the three main components:

- $f(\mu)$ is the prior distribution of the parameter of interest for inference, $\mu$ : it summarizes the initial subjective opinion detained by the decision-maker about the probability density function of the parameter $\mu$;

- $L\left(\mu ; y_{1}, y_{2}, \ldots, y_{n}\right)$ is the likelihood function, obtained after the collection of the $n$ experimental data $y_{1}, y_{2}, \ldots, y_{n}$

- $f\left(\mu \mid y_{1}, y_{2}, \ldots, y_{n}\right)$ is the posterior distribution, i.e. the distribution that expresses the knowledge acquired about the parameter $\mu$ after updating the initial subjective judgments with the experimental data.

In summary, the Bayesian approach allows us to translate tacit knowledge into probabilistic distributions and, secondly, to integrate tacit knowledge and data records in the forecasting process.

\section{A Bayesian model for cost and time estimates at completion}

As mentioned above, the EVM methodology provides parameters and performance indexes which are commonly used in project control. In the Oil \& Gas examples, a Bayesian model has been applied, where two performance indexes are used:

$N D(c)_{T N}=\frac{A C_{T N}-E V_{T N}}{E V_{T N}}$

$N D(t)_{T N}=\frac{T N-E S_{T N}}{E S_{T N}}$

where $N D(c)_{T N}$ stands for Normalized Deviation (cost) at the time now and similarly $N D(t)_{T N}$; when referring to the performance indicator in general, without distinction between time and cost, the acronym ND, Normalized Deviation, will be used.

In the Bayesian model, the parameter of inference is the Normalized Deviation of cost and time evaluated at completion, identified by the equations (6) and (7): 
$N D(c)=\frac{A C_{T F}-B A C}{B A C}$

$N D(t)=\frac{T F-S A C}{S A C}$

where $\mathrm{AC}_{\mathrm{TF}}$ and TF indicate the final actual cost and duration of the project, while BAC and SAC indicate the planned cost and duration of the project, respectively. When a budget overrun is reported, ND is greater than zero, whereas if an under-run is recorded, ND is less than zero. In the following sections, the model will be discussed in three main parts, following the three basic components of the Bayes theorem: the prior distribution, the likelihood function and the posterior distribution. In order to make reading easier, the formulas will not be extensively demonstrated, however the detailed proofs of the final expressions can be found in the Appendix.

\subsection{Prior distribution}

In the Bayes theorem, the prior distribution is a summary of the initial knowledge that the decisionmaker has available for the specific parameter of interest. In the case of projects, the project team can rely on two main information sources: experts' opinions and data records from similar projects completed in the past. By integrating these two components, a starting assumption about the distribution of $N D_{T F}$ can be formulated. In order to translate these concepts in Bayesian terms, firstly two distributions have to be defined: one representing the experts' opinions and one the cluster of similar projects. Secondly, a unique distribution has to be derived by the integration of these two. In mathematical terms, the integration can be obtained by the concept of mixture: assigning a value ranging from 0 to 1 to a weight $\varepsilon$, the prior distribution is obtained as the weighted sum of the distribution of experts' opinions and the distribution of the cluster of similar projects:

$\Pi(\cdot)=\varepsilon \Pi_{E}(\cdot)+(1-\varepsilon) \Pi_{S}(\cdot)$

In Equation (8):

- . is a generic point of the prior distribution; 
- $\Pi_{E}(\cdot)$ is the evaluation of the distribution of experts' opinions in a generic point $\cdot ;$

- $\Pi_{S}(\cdot)$ is the evaluation of the distribution of the cluster of similar projects in a generic point $\cdot ;$

- $\varepsilon$ is the weight.

Adjusting the value assigned to $\varepsilon$ it is possible to give more or less weighting to one of the two informative sources. Moreover, even if one of the two sources is lacking, it is still possible to define the prior distribution setting the value of the weight to 0 or 1: 0 if no experts can be identified and 1 if no similar project is available. Consequently, the prior distribution is characterized by flexibility, since it can be formulated according to the sources of information available to the project team.

From Eq. (8) it clearly appears that the shape assumed by the prior distribution depends on the two distributions of experts and similar projects and on the value assigned to the weight $\varepsilon$. In the following sections the distribution of the experts' opinions and the similar projects will be defined and guidelines will be given about the value of $\varepsilon$.

\subsection{Distribution of experts' opinions}

The starting assumption for the experts' opinions is that they can be expressed through a normal distribution of the variable that is analyzed. This assumption has to be verified when applying the model. Hence, in order to completely determine the distribution, two main parameters have to be identified: the mean and the variance $\left(\mu_{E}\right.$ and $\left.\sigma_{E}^{2}\right)$. In order to define them, a process to elicit the information has to be performed with the aim of obtaining a distribution consistent with the knowledge owned by the group of experts involved. The main obstacle is the above mentioned (see section 2) cognitive and motivational bias which tends to create a gap between the actual knowledge available and the opinions expressed.

As a consequence, the proposed procedure for eliciting experts' knowledge is based on the Analytic Hierarchy Process (AHP) methodology, since through pair-wise comparisons a more robust result can be obtained, compared with other methodologies based on a direct estimate of the parameter being analyzed. AHP has been used to support the fixed interval method. According to this method, in order to elicit the distribution of a certain parameter $X$, the domain of definition of $X$ has to be subdivided into intervals and each interviewee expresses his preference for a specified interval 
through a pair-wise comparison between the intervals. Otherwise, if the interviewee would be directly asked to give probability values to the intervals, the cognitive and motivational bias would certainly bias the final result (Saaty, 1994; Forman and Peniwati, 1998).

In our case, the alternatives are represented by the intervals of the domain of NDTF and the criterion applied is only that the preference between two intervals will be expressed on the basis of the corresponding probability levels. In summary, the main advantages of adopting AHP is the robustness of the end results, the adaptability of the methodology to a single expert or a group of experts and the possibility to control the consistency of the elicited opinions during the application of the technique.

\subsection{Distribution of similar projects}

The second component of the prior distribution is the distribution of the cluster of projects which are similar to the ongoing project. The role of this cluster is to exploit past experience in order to reduce possible bias, e.g. over-optimism that typically may affect the experts' opinions at the project outset (Flyvberg, 2006). Even in this case, the assumption - which has to be verified - is that the set of data has a normal distribution.

Before entering into the details of the similarity analysis, Eq. (9) and Eq. (10) show how to evaluate the parameters of the distribution, $\mu_{\mathrm{s}}$ and $\sigma_{\mathrm{s}}{ }^{2}$, of the cluster of similar projects, considering $n$ projects with actual cost or time deviation from planned $N D_{T F i}$ :

$$
\begin{gathered}
\boldsymbol{\mu}_{\boldsymbol{S}}=\frac{\sum_{i=1}^{n} \boldsymbol{N} \boldsymbol{D}_{T F, i}}{n} \\
\sigma_{S}=\sqrt{\frac{\sum_{i=1}^{n}\left(N D_{T F, i}-\widehat{\mu_{S}}\right)^{2}}{n-1}}
\end{gathered}
$$

This approach safeguards the subjectivity of the similarity analysis, confirming the subjective nature of the overall prior distribution. In order to validate the model in the three Oil\&Gas case studies the following similarity criteria have been used.

Firstly, of the projects in the database, only those with a scope of work similar to the ongoing project have been considered for the similarity analysis. For example, if the ongoing project aims at building 
an onshore facility, only the onshore projects have been taken into account. From this point of view, three types of project have been considered: on shore, off shore and subsea.

Secondly, the similarity between the ongoing project and those identified at the previous step has been analyzed. The criteria applied for this purpose envision, besides the traditional project features, such as size, technology, client, geographical area, etc., even the project risk profile. The assumption is that risk profile - risk quantity, risk type, risk exposure, etc. - is an effective factor to explain a similar development of two different projects in terms of the deviation from planned budget and schedule. After selecting the cluster of similar projects, $\boldsymbol{\mu}_{\boldsymbol{S}}$ and $\boldsymbol{\sigma}_{\boldsymbol{S}}$ can be estimated applying Eq. (9) and Eq. (10), respectively.

\subsection{Definition of the weight $\varepsilon$}

The last parameter to be defined in order to identify the prior distribution is the weight $\varepsilon$. When $\varepsilon$ increases, the weight of the experts' distribution increases, while the weight of the distribution of similar projects decreases.

Recalling figure 1, a first observation should point out that experts' opinions tend to be more accurate as the project progress increases. Therefore, the initial value assigned to the weight must be increased as the project approaches the end. Indicating with the letter $k$ the progress reached by the ongoing project at Time Now, in decimals (for example $0.2,0.15,0.8$ ), a possible range for the value assigned to the weight $\varepsilon$ is expressed by the Eq. (11):

$\varepsilon=\in[\mathbf{0}, \mathbf{1}]$

Performing a sensitivity analysis with respect to changes in $\varepsilon$ is a good statistical practice, since the decision maker is, in general, unable to express a precise value of $\varepsilon$. Therefore, $\varepsilon$ is varied by small intervals around the elicited value, expressing the decision maker's uncertainty, and the changes on the final decision value are examined. Should the latter be almost unchanged, then the procedure could go ahead since imprecision on the assessment of $\varepsilon$ would have minimal effect in practice; 
otherwise the decision maker would be asked for more information in order to get a more precise value of $\varepsilon$.

\subsection{Prior distribution}

After the identification of the parameters $\mu_{\mathrm{E}}, \sigma_{\mathrm{E}}$ and $\mu_{\mathrm{S}}, \sigma_{\mathrm{S}}$ and the assignment of a value to the weight $\varepsilon$, the prior distribution is completely defined. From Eq. (6), the prior distribution is given by:

$\Pi(x)=\varepsilon \cdot \frac{1}{\sqrt{2 \pi} \sigma_{E}} e^{-\frac{1}{2}\left(\frac{x-\mu_{E}}{\sigma_{E}}\right)^{2}}+(1-\varepsilon) \cdot \frac{1}{\sqrt{2 \pi} \sigma_{S}} e^{-\frac{1}{2}\left(\frac{x-\mu_{S}}{\sigma_{S}}\right)^{2}}$

Hence, the prior distribution is a mixture of two normal distributions. The shape it assumes may vary and, for instance, three different types of shape can be obtained:

- normal;

- skewed normal;

- bimodal.

Obviously, the shape will depend on the five parameters which characterize the prior distribution. The variety of shapes denotes the flexibility of the prior distribution defined as a mixture of two contributions. The prior distribution is not caged inside a pre-defined shape, on the contrary, adjusting $\varepsilon$, the decision-maker can obtain the shape that better fits his/her opinions.

\subsection{Likelihood function}

The starting assumption is that $N D(c)_{T N}$ and $N D(t)_{T N}$ have a normal distribution. The experimental data collection will be represented by a single observation of the performance index, i.e. $N D(c)_{T N}$ and $N D(t)_{T N}$. These are cumulated indicators and, therefore, their value sums up the entire interval from the project start up to the Time Now.

The normality assumption can be justified considering that, generally speaking, the observation at time now can differ from the unknown final value $N D_{T F}$ by a positive or negative quantity: the relationship between the final unknown performance and the one registered at the Time Now can be expressed as follows:

$x(t)=\mu+\sigma(t) \cdot e$ 
In Equation (13), $x$ is the observation at time now, $\mu$ is the final unknown parameter, $\sigma(t)$ is the standard deviation and $e$ is the error that, in the case of a normal likelihood function, is standardnormally distributed i.e. $e \sim N(0,1)$.

The second assumption is that the standard deviation of the likelihood function $\sigma(t)$ is known. In order to determine its value the meaning of $\sigma(t)$ has to be clarified. From Eq. (13), it can be noticed that $\sigma(t)^{-1}$ quantifies the degree of confidence that can be attributed to the observation at time now. In other words, the more the observation at time now can be reasonably assumed to be close to the final actual value, the lower will be the value to be assigned to the $\sigma$ of the likelihood function.

Thus, two conclusions can be deduced. First, as the project progress increases, the standard deviation is reduced since the performance at the time now will be closer and closer to the final value. This statement can be explained by the cumulative nature of the performance indexes. Second, the higher is the difference between the observation at time now and the expected value of the prior distribution, the less trust-worthy is the observation at time now and the larger the standard deviation. In fact, the prior distribution may be considered a valid forecast for the final performance index, since it matches two informative sources.

Starting from these remarks, two corresponding indicators can be defined: a progress and a distance indicator. The progress indicator can assume three qualitative values: low, medium and high, corresponding to the physical progress reached by the ongoing project at Time Now. The distance indicator can also assume a low, medium or high value depending on the difference between the observation at time now and the expected value of the prior distribution which summarizes the prior opinion (Eq. (12) and Eq. (13)):

$\Delta_{c}=\left|N D(c)_{T N}-E_{p}\left[N D(c)_{T F}\right]\right|$

$\Delta_{T}=\left|N D(t)_{T N}-E_{p}\left[N D(t)_{T F}\right]\right| \cdot S A C$

$\Delta_{c}$ refers to the "cost distance" and $\Delta_{T}$ to the "time distance". $E_{p}\left[N D(c)_{T F}\right]$ is the expected value of the prior distribution of the final cost performance and $E_{p}\left[N D(t)_{T F}\right]$ of the final time performance. 
SAC is the Schedule at Completion: if it is expressed in months, it provides the delta in months. In Table 1, the classes for the distance indicator and the project progress indicator are defined:

\begin{tabular}{|c|c|c|}
\hline \multicolumn{3}{|c|}{ Project Progress } \\
\hline Low & Medium & High \\
\hline$[0,30 \%)$ & {$[30 \%, 65 \%]$} & $(65 \%, 100 \%]$ \\
\hline \multicolumn{3}{|c|}{$\Delta_{C}$} \\
\hline Low & Medium & High \\
\hline$[0,0.05]$ & $(0.05,0.1]$ & $>0.1$ \\
& \multicolumn{2}{|c|}{$\Delta_{T}$} \\
\hline & & \\
\hline Low & Medium & High \\
\hline [0, 3 months $)$ & {$[3,5$ months $)$} & $\geq 5$ months \\
\hline
\end{tabular}

Table 1. Progress and Distance Classes

When the class (low, medium, high) of project progress and of distance at the Time Now has been identified, a corresponding value can be assigned to the standard deviation thanks to the following matrix in Table 2:

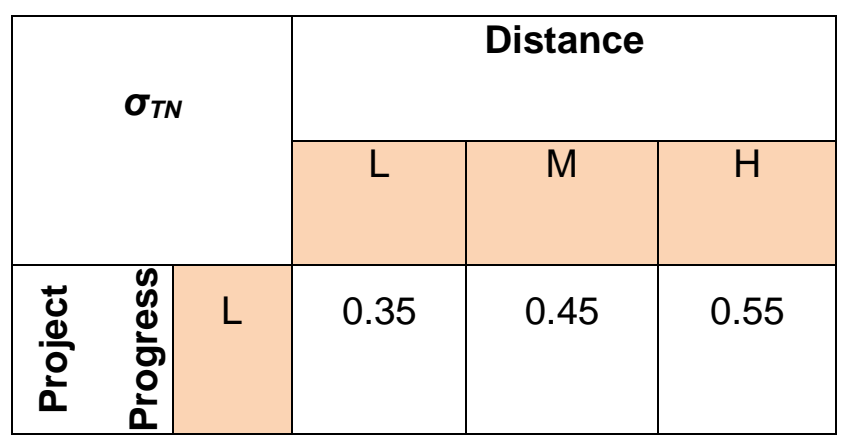




\begin{tabular}{|l|c|c|c|c|}
\hline & $M$ & 0.25 & 0.35 & 0.45 \\
\cline { 2 - 5 } & $\mathrm{H}$ & 0.15 & 0.25 & 0.35 \\
\hline
\end{tabular}

Table 2. Standard deviation for the likelihood function

The standard deviation has been assumed to range from 0.15 to 0.55 , increasing as the distance increases and as the project progress decreases. After having evaluated the performance index at Time Now and the standard deviation from Tab.2, the likelihood function is completely defined. Indicating $\mathrm{ND}(\mathrm{c})_{\mathrm{TN}}$ or $\mathrm{ND}(\mathrm{t})_{\mathrm{TN}}$ with $\mathrm{x}$ and the parameter of inference with $\boldsymbol{\mu}$, the expression of the likelihood function is given in Eq. (16):

$L_{x}(\mu)=\frac{1}{\sqrt{2 \pi} \sigma} \cdot \exp \left[-\frac{1}{2}\left(\frac{x-\mu}{\sigma}\right)^{2}\right]$

\subsection{Posterior distribution}

Combining the prior distribution in Eq. (12) and the likelihood function in Eq. (16) according to the Bayes theorem in Eq. (1), the following posterior distribution can be obtained:

$\Pi(\mu \mid x)=\left(1-\varepsilon^{*}\right) \cdot \Pi_{S}(\mu \mid x)+\varepsilon^{*} \cdot \Pi_{E}(\mu \mid x)$

The posterior function is a mixture of the posterior distribution of similar projects $\Pi_{S}(\mu \mid x)$ and the posterior distribution of experts' opinions $\Pi_{E}(\mu \mid x)$ through the posterior weight $\varepsilon^{*}$. These three components are defined in Eq. (18), Eq. (19) and Eq. (20).

$\Pi_{S}(\mu \mid x): N\left(\frac{\sigma^{2} \cdot \mu_{S}+\sigma_{S}^{2} \cdot x}{\sigma^{2}+\sigma_{S}^{2}}, \frac{\sigma^{2} \cdot \sigma_{S}^{2}}{\sigma^{2}+\sigma_{S}^{2}}\right)$ 
$\Pi_{E}(\mu \mid x): N\left(\frac{\sigma^{2} \cdot \mu_{S}+\sigma_{E}^{2} \cdot x}{\sigma^{2}+\sigma_{E}^{2}}, \frac{\sigma^{2} \cdot \sigma_{E}^{2}}{\sigma^{2}+\sigma_{E}^{2}}\right)$

$\varepsilon^{*}=\frac{\varepsilon \cdot D_{E}}{(1-\varepsilon) \cdot D_{S}+\varepsilon \cdot D_{E}}$

Eq.(21) and Eq.(22) clarify the expression of $D_{E}$ and $D_{S}$, that are useful to calculate the posterior weight $\varepsilon^{*}$.

$D_{E}=\frac{1}{\sqrt{2 \pi\left(\sigma_{E}{ }^{2}+\sigma^{2}\right)}} \cdot e^{-\frac{1}{2}\left(\frac{x-\mu_{E}}{\sqrt{\left(\sigma_{E}{ }^{2}+\sigma^{2}\right)}}\right)^{2}}$

$D_{S}=\frac{1}{\sqrt{2 \pi\left(\sigma_{S}^{2}+\sigma^{2}\right)}} \cdot e^{-\frac{1}{2}\left(\frac{x-\mu_{S}}{\sqrt{\left(\sigma_{S}^{2}+\sigma^{2}\right)}}\right)^{2}}$

Finally, the expression of variance and standard deviation of the posterior distribution can be evaluated through Eq. (23) and Eq. (24):

$E(\mu \mid x)=\left(1-\varepsilon^{*}\right) \cdot E_{S}(\mu \mid x)+\varepsilon^{*} \cdot E_{E}(\mu \mid x)$

$\operatorname{Var}(\mu \mid x)=E\left(\mu^{2} \mid x\right)-[E(\mu \mid x)]^{2}$

$E\left(\mu^{2} \mid x\right)=\left(1-\varepsilon^{*}\right) \cdot\left\{\sigma^{2}{ }_{S}(\mu \mid x)+E_{s}\left(\mu^{2} \mid x\right)\right\}+\varepsilon^{*} \cdot\left\{\sigma_{E}^{2}(\mu \mid x)+E_{E}\left(\mu^{2} \mid x\right)\right\}$

where:

- $E_{E}(\mu \mid x)$ is the expected value of experts' posterior distribution : $\frac{\sigma^{2} \cdot \mu_{E}+\sigma_{E}^{2} x}{\sigma^{2}+\sigma_{E}{ }^{2}}$. 
- $E_{S}(\mu \mid x)$ is the expected value of similar projects' posterior distribution: $\frac{\sigma^{2} \cdot \mu_{S}+\sigma_{S}^{2} \cdot x}{\sigma^{2}+\sigma_{S}{ }^{2}}$.

- $\sigma_{E}^{2}(\mu \mid x)$ is the variance of experts' posterior distribution: $\frac{\sigma^{2} \cdot \sigma_{E}^{2}}{\sigma^{2}+\sigma_{E}^{2}}$.

- $\sigma_{S}^{2}(\mu \mid x)$ is the variance of similar projects' posterior distribution: $\frac{\sigma^{2} \cdot \sigma_{S}^{2}}{\sigma^{2}+\sigma_{S}^{2}}$.

\section{Case studies: application of the Bayesian model to three Oil and Gas megaprojects}

The research activity was developed in the years 2013-2014. The largest Italian company in the Oil\&Gas industry was involved. In this industry, projects are very large, complex and uncertain, therefore the forecasting process is very critical. Three types of projects have been identified: on shore, off shore and subsea. Offshore projects are denoted by offshore facilities for drilling and extracting hydrocarbons. Fixed or floating platforms can be used, depending on water depth. The extracted hydrocarbons are then transported onshore through a sea line-system.

Onshore projects are characterized by the construction and installation of onshore facilities. The liquid and/or gas hydrocarbons extracted from the wells are firstly stored and then the liquids are sent through flow-lines to a preliminary treatment unit and then to a refinery. The gas follows a similar process until the preliminary treatment; afterwards it is sent for further treatment through a gas pipeline.

Subsea projects are characterized by undersea facilities for extraction and production. The need to install undersea facilities is due to the technical or economical unfeasibility of utilizing offshore platforms. When there are more than one undersea wells, the wells are interconnected through flow lines, in addition to the sea-lines that link the wells of extraction and production with onshore facilities.

Based on this classification, three clusters of about 6 to 8 projects completed in the past have been identified and used as classes of reference. For each cluster one of the most recent projects was chosen as ongoing project. At different times (Time Now) throughout the life cycle of the ongoing project the forecasting process was simulated, based only on the information available at that Time Now, both in terms of data records and experts' judgment. 
Whilst data records were immediately available from the corporate information system, experts' judgments had to be collected through a set of interviews. The chosen interviewees were five project managers not directly involved in the three ongoing projects, each featuring a different level of professional experience. The interviews have been carried out using the same methodology, whilst a different weight has been assigned to each expert, based on the years of experience in the Oil and Gas industry.

For each project, each interviewee was asked to simulate a forecasting process at each Time Now throughout the project life cycle, using only the available knowledge at the Time Now. In order to guarantee that the process for obtaining the experts' opinions was robust and reliable, the Analytic Hierarchy Process (AHP) was adopted as an eliciting approach, based on a set of pairwise comparison between the alternatives considered during the estimating process.

For every pairwise comparison, the same question has been asked to the interviewees, i.e.: "How probable is that the final time / budget deviation will fall in the first interval, respect to the second one?". The answer expressed in the natural language were then converted into numbers ranging from 1 to 9 .

After the elicitation round, before evaluating the overall probability for each interval and fitting the corresponding distribution, the consistency of the values obtained from each expert has been verified, using the classic Consistency Ratio provided by the AHP theory. In case the consistency ratio is above 0.1 , the matrix would result to be inconsistent. In this case the software Expert Choice points out the intervals to be revised.

Finally, the judgments expressed by the single experts have to be summarized in a group's matrix. Forman and Peniwati (1997) suggest to use the geometric mean of the judgements, related to each couple of intervals. Through AHP methodology (Saaty, 1994) the final set of probability values assigned to each interval can be obtained, i.e. the probability that the pool of experts has decided to assign to each interval. Lastly, one distribution, assumed to be normal, can be defined both for budget overrun $\mathrm{ND}$ and completion delay $\mathrm{ND}(\mathrm{t})$, by evaluating expected value and standard deviation. 
The Bayesian model has been applied to three projects, each of them belonging to one of the three types of project. The Offshore project will be named Project A, the Subsea one as Project B and the Onshore one as Project C.

The scope of work of project A consists of the installation of a fixed conventional platform 100 miles from the coast and in waters of $60 \mathrm{~m}$ depth. There are six wells to be drilled and a sea-pipeline has to be set up as well in order to connect the different wells to the platform.

\begin{tabular}{|c|c|}
\hline \multicolumn{2}{|c|}{ Project A: Offshore } \\
\hline $\begin{array}{l}\text { New/Legacy } \\
\text { Area }\end{array}$ & Legacy Area \\
\hline$B A C$ & 231.26 MUSD \\
\hline$S A C$ & 30 months \\
\hline Time Now & Month 16 \\
\hline \multicolumn{2}{|c|}{ Planned Data } \\
\hline$P V_{T N}$ & 205.82 MUSD \\
\hline$P P_{T N}$ & $51.13 \%$ \\
\hline \multicolumn{2}{|c|}{ Actual Data } \\
\hline$A C_{T N}$ & 103.82 MUSD \\
\hline$A P_{T N}$ & $36.45 \%$ \\
\hline \multicolumn{2}{|c|}{ Earned Value and Earned Schedule } \\
\hline$E V_{T N}$ & 97.52 MUSD \\
\hline
\end{tabular}




\begin{tabular}{|l|c|}
\hline$E S_{T N}$ & 13.56 months \\
\hline \multicolumn{2}{|c|}{ Time Now Performances } \\
\hline$N D(c)_{T N}$ & $6.46 \%$ \\
& $17.99 \%$ \\
\hline$N D(t)_{T N}$ & \\
\hline
\end{tabular}

Table 3. Project A-Offshore

Basic data about Project A are defined in Table 3, where:

- $\quad P P_{T N}$ stands for Planned Progress at Time Now, in agreement with the baseline;

- $A P_{T N}$ is the Actual Progress at Time Now, i.e. the physical actual progress reached by the project at Time Now.

The first row in Table 3 "New/Legacy Area" indicates whether the project is developed in a geographical area where the company has experience in operating or in a New Area. In the case of a New Area the degree of uncertainty of the project rises, since there is no established relationship with suppliers and subcontractors. In particular, the $A P_{T N}$ of $36.45 \%$ is to be noted, since it will be useful to define the weights of the prior distribution and the standard deviation of the likelihood function.

Project $B$ is a subsea project and has the following scope of work:

- drilling of three undersea wells.

- set up of a FPSO (Floating Production Storage and Offloading) plant;

- installation of flexible flow-lines to interconnect the three wells and of an umbilical to transport the extracted oil to the FPSO.

From Table 4. we can see that Project B is being developed in a New Area, implying the issues previously discussed. The $\mathrm{AP}_{\mathrm{TN}}$ amounts to $14.54 \%$. Actually, the project is on schedule but a cost underrun of approximately $60 \%$ has been recorded. 


\begin{tabular}{|c|c|}
\hline \multicolumn{2}{|c|}{ Project B: Subsea } \\
\hline New/Legacy Area & New Area \\
\hline$B A C$ & 314.93 MUSD \\
\hline$S A C$ & 32 months \\
\hline Time Now & Month 13 \\
\hline \multicolumn{2}{|c|}{ Planned Data } \\
\hline$P V_{T N}$ & 81.92 MUSD \\
\hline$P P_{T N}$ & $14.87 \%$ \\
\hline \multicolumn{2}{|c|}{ Actual Data } \\
\hline$A C_{T N}$ & $32.60 \mathrm{MUSD}$ \\
\hline$A P_{T N}$ & $14.54 \%$ \\
\hline \multicolumn{2}{|c|}{ Earned Value and Earned Schedule } \\
\hline$E V_{T N}$ & 80.63 MUSD \\
\hline$E S_{T N}$ & 12.87 months \\
\hline \multicolumn{2}{|c|}{ Time Now Performances } \\
\hline$N D(c)_{T N}$ & $-59.56 \%$ \\
\hline$N D(t)_{T N}$ & $1.01 \%$ \\
\hline
\end{tabular}

Table 4. Project B-Subsea

The last case study is an Onshore project. The scope of work entails: 
- perforation of 13 new wells and re-entry in 10 pre-existing wells;

- installation of 23 flowlines;

- installation of an Onshore facility to process the extracted hydrocarbons;

- installation of a system to export the extracted hydrocarbons.

\begin{tabular}{|c|c|}
\hline \multicolumn{2}{|c|}{ Project C: Onshore } \\
\hline New/Legacy Area & Legacy Area \\
\hline$B A C$ & 1500 MUSD \\
\hline SAC & 37 months \\
\hline Time Now & Month 26 \\
\hline \multicolumn{2}{|c|}{ Planned Data } \\
\hline$P V_{T N}$ & 1265 MUSD \\
\hline$P P_{T N}$ & $79.22 \%$ \\
\hline \multicolumn{2}{|c|}{ Actual Data } \\
\hline$A C_{T N}$ & 1094 MUSD \\
\hline$A P_{T N}$ & $76.4 \%$ \\
\hline \multicolumn{2}{|c|}{ Earned Value and Earned Schedule } \\
\hline$E V_{T N}$ & 1072.63 MUSD \\
\hline$E S_{T N}$ & 25.47 months \\
\hline \multicolumn{2}{|c|}{ Time Now Performances } \\
\hline$N D(c)_{T N}$ & $1.99 \%$ \\
\hline
\end{tabular}




\begin{tabular}{|l|c|}
\hline$N D(t)_{T N}$ & $2.08 \%$ \\
& \\
\hline
\end{tabular}

Table 5. Project C-On-shore

Note the high $\mathrm{AP}_{\mathrm{TN}}(76.4 \%)$ and the good budget and schedule performance at Time Now.

\subsection{Prior distributions}

In Fig. 3 the prior distributions for the final cost and time performance of Project $A$ are reported. The forecast of the experts is more optimistic than the one deriving from the cluster of similar projects, both for time and cost final performance. The two prior distributions are symmetrical with respect to the mode and, since $\varepsilon=0.2$, the most relevant contribution to the parameters of the prior distributions is from the distribution of the cluster of similar projects. This statement can be verified in Table 6 .

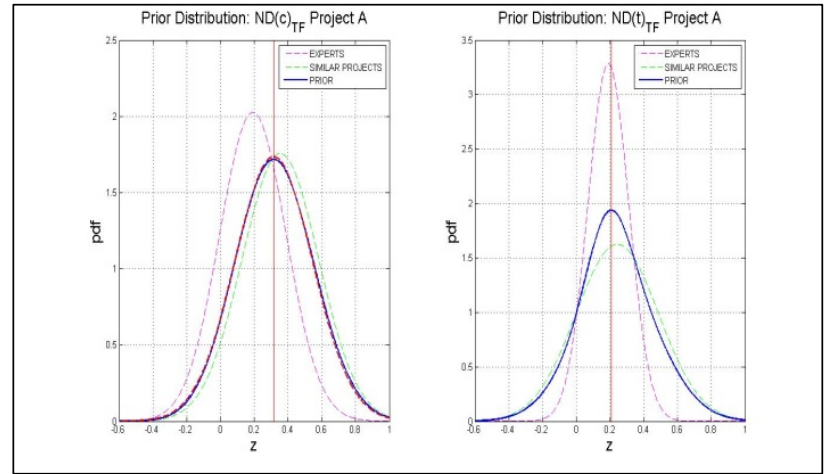

Figure 3. Prior Distribution Project A

\begin{tabular}{|l|c|c|c|c|}
\hline \multicolumn{5}{|c|}{ Prior Distribution Project A, $\varepsilon=0.2$} \\
\hline \multirow{2}{*}{} & \multicolumn{2}{|c|}{ ND(c) TF } & \multicolumn{2}{c|}{ ND(t) TF } \\
\hline Similar & $\mu_{S}$ & $\sigma_{S}$ & $\mu_{S}$ & $\sigma_{S}$ \\
Projects & 0.3547 & 0.2271 & 0.2428 & 0.2455 \\
\cline { 2 - 5 } & & & & \\
\hline
\end{tabular}




\begin{tabular}{|l|l|l|l|l|}
\hline \multirow{4}{*}{ Experts } & \multicolumn{1}{|c|}{$\mu_{E}$} & \multicolumn{1}{|c|}{$\sigma_{E}$} & \multicolumn{1}{|c|}{$\mu_{E}$} & \multicolumn{1}{|c|}{$\sigma_{E}$} \\
\cline { 2 - 5 } & 0.1917 & 0.1971 & 0.1899 & 0.1215 \\
\hline \multirow{3}{*}{ Prior } & Ealue & Dev. & Value & Dev. \\
\cline { 2 - 5 } & 0.3222 & 0.2308 & 0.2322 & 0.2272 \\
\hline
\end{tabular}

Table 6. Composition of Prior Distribution Project A-Offshore

Fig.4 clearly shows that the prior distribution of $N D(c)_{T F}$ is asymmetric to the left. As a matter of fact, the distribution of the experts' opinions is much more optimistic than the one provided by the cluster of similar projects.

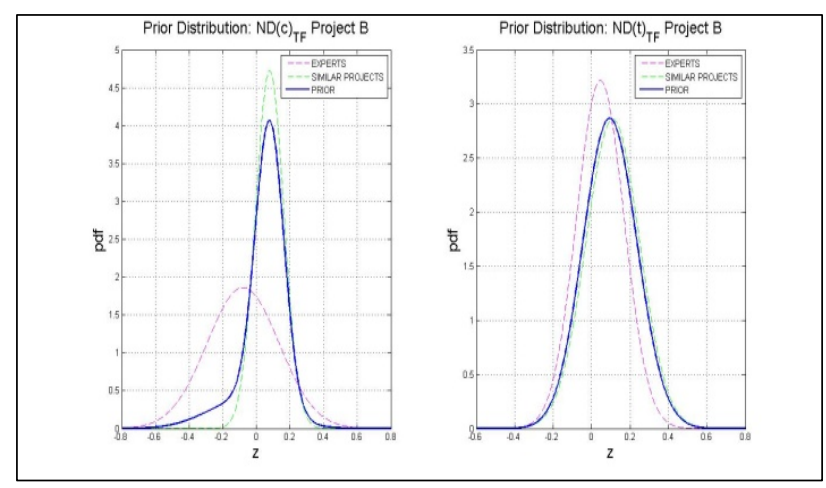

Figure 4. Prior Distribution Project B

Considering that $N D(c)_{T N}=-0.5956$, i.e. a $60 \%$ budget underrun is reported, the group of experts forecasted that by the end of the project the expected value of the budget deviation is around $-8 \%$ : there will be in other words a slight budget underrun ( $\mu_{E}$ for $N D(c)_{T F}$ equals -0.0793 , as shown in Table 7). The skewness is caused by the different dispersion of experts' opinions compared to the the cluster of similar projects: the standard deviation of experts' distribution is much higher. 
As far as schedule performance is concerned, even in this case the experts were just slightly more optimistic than the statistics provided by the cluster of similar projects, but the prior distribution of $N D(t)_{T F}$ is almost symmetrical because of the similar standard deviation of the two contributions.

In conclusion it is important to note that the weight attributed to the experts' distribution is 0.2 because of the low physical progress reached at time now (14.5\%).

\begin{tabular}{|c|c|c|c|c|}
\hline \multicolumn{5}{|c|}{ Prior Distribution Project $B, \varepsilon=0.2$} \\
\hline & \multicolumn{2}{|c|}{$\mathrm{ND}(\mathrm{c})_{\mathrm{TF}}$} & \multicolumn{2}{|c|}{$N D(t)_{T F}$} \\
\hline \multirow{2}{*}{$\begin{array}{l}\text { Similar } \\
\text { Projects }\end{array}$} & $\mu_{S}$ & $\sigma_{S}$ & $\mu_{S}$ & $\sigma_{S}$ \\
\hline & 0.0792 & 0.0844 & 0.11 & 0.1395 \\
\hline \multirow[b]{2}{*}{ Experts } & $\mu_{E}$ & $\sigma_{E}$ & $\mu_{E}$ & $\overline{\sigma_{E}}$ \\
\hline & -0.0793 & 0.214 & 0.0455 & 0.1248 \\
\hline \multirow[t]{2}{*}{ Prior } & $\begin{array}{l}\text { Expected } \\
\text { Value }\end{array}$ & $\begin{array}{l}\text { Standard } \\
\text { Dev. }\end{array}$ & $\begin{array}{l}\text { Expected } \\
\text { Value }\end{array}$ & $\begin{array}{l}\text { Standard } \\
\text { Dev. }\end{array}$ \\
\hline & 0.0476 & 0.1373 & 0.0970 & 0.1390 \\
\hline
\end{tabular}

Table 7. Composition of Prior Distribution Project B-Subsea

In the case of Project $C$, it has been decided to assign $\varepsilon=0.8$ because of the high physical progress reached at time now which is equal to $76.4 \%$. The experts' forecast for $N D(c)_{T F}$ appears more pessimistic than the one provided by the cluster of similar projects even though the dispersion is similar; hence, this prior distribution is symmetrical, as shown in Fig.5, left. 


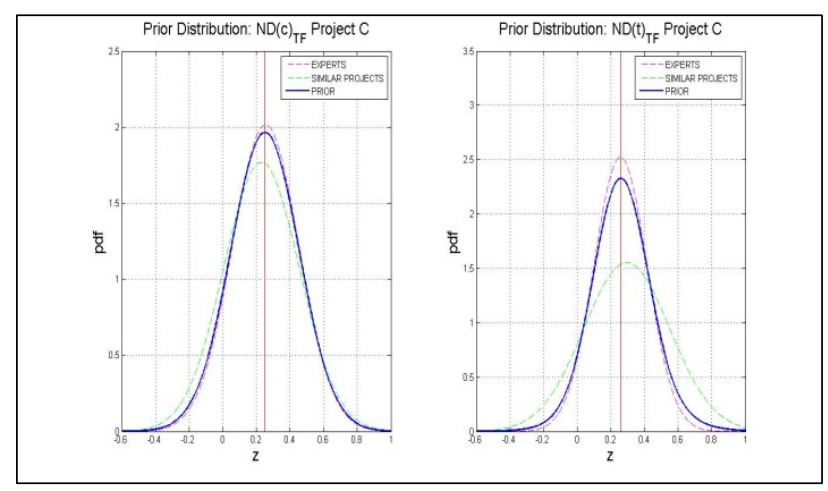

Figure 5. Prior Distribution Project C

\begin{tabular}{|c|c|c|c|c|}
\hline \multicolumn{5}{|c|}{ Prior Distribution Project C, $\varepsilon=0.8$} \\
\hline & \multicolumn{2}{|c|}{$\mathrm{ND}(\mathrm{c})_{\mathrm{TF}}$} & \multicolumn{2}{|c|}{$\mathrm{ND}(\mathrm{t})_{\mathrm{TF}}$} \\
\hline \multirow{2}{*}{$\begin{array}{l}\text { Similar } \\
\text { Projects }\end{array}$} & $\mu_{S}$ & $\sigma_{S}$ & $\mu_{S}$ & $\sigma_{S}$ \\
\hline & 0.2307 & 0.2255 & 0.2948 & 0.2569 \\
\hline \multirow[b]{2}{*}{ Experts } & $\mu_{E}$ & $\sigma_{E}$ & $\mu_{E}$ & $\sigma_{E}$ \\
\hline & 0.2552 & 0.1983 & 0.2572 & 0.158 \\
\hline \multirow{2}{*}{ Prior } & $\begin{array}{c}\text { Expected } \\
\text { Value }\end{array}$ & $\begin{array}{c}\text { Standard } \\
\text { Dev. }\end{array}$ & $\begin{array}{c}\text { Expected } \\
\text { Value }\end{array}$ & $\begin{array}{c}\text { Standard } \\
\text { Dev. }\end{array}$ \\
\hline & 0.2501 & 0.2040 & 0.2646 & 0.1828 \\
\hline
\end{tabular}

Table 8. Composition of Prior Distribution Project C

The experts' forecast for $\mathrm{ND}(\mathrm{t})_{\mathrm{TF}}$ is more optimistic and the confidence in the current schedule performance is higher ( $\sigma_{E}$ equals 0.158 , while $\sigma_{S}$ is 0.2529 , Tab.8). The result is still a symmetrical prior distribution (Fig.6, right). Finally, it is important to note that, even though at time now $N D(c)_{T N}$ 
amounts at $1.99 \%$ and $N D(t)_{T N}$ equals to $2.08 \%$ (see Table 8 ), the prior distribution forecasts a high probability of a worsening of time and cost performance, despite the actual physical progress reached at time now. In fact, it may be considered that, since the actual progress at time now is almost 80\%, the actual final performance will not largely differ from the one at time now; however, in the Oil and Gas industry many stakeholders are involved and political issues and causes of force majeure (conflicts, weather instability, etc.) can suddenly intervene causing a drastic worsening of time and cost performances.

\subsection{Posterior Distributions}

Tale 9 shows that the parameters of the posterior distributions of $N D(c)_{T F}$ and $N D(t)_{T F}$ are obtained through the combination of the prior distribution and the likelihood function. In particular, the standard deviation of the distributions decreases from the prior to the posterior distribution. In fact, the final distribution is the result of the integration of more information sources and hence the confidence level about the expected value of the final distribution is consequently higher.

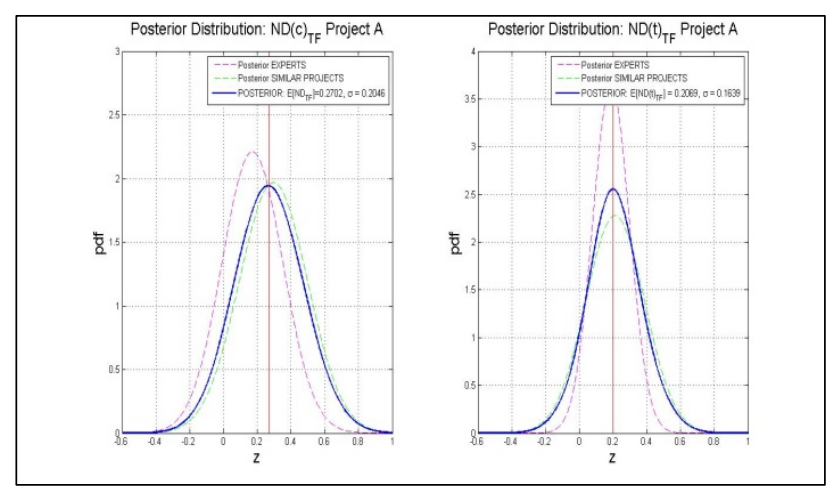

Figure 6. Posterior Distribution Project A

\begin{tabular}{|l|l|l|}
\hline \multicolumn{2}{|c|}{ Posterior Distribution Project $A, \varepsilon=0.2$} \\
\hline & $\mathrm{ND}(\mathrm{c})_{\mathrm{TF}}$ & $\mathrm{ND}(\mathrm{t})_{\mathrm{TF}}$ \\
\hline
\end{tabular}




\begin{tabular}{|l|c|c|c|c|}
\hline Prior & Expect. & Stand. & Expect. & Stand. \\
& Val. & Dev. & Val. & Dev. \\
\cline { 2 - 5 } & 0.3222 & 0.2308 & 0.2322 & 0.2272 \\
\hline Likelihood & $N D(c)_{T N}$ & $\sigma_{T N}$ & $N D(t)_{T N}$ & $\sigma_{T N}$ \\
& & & & \\
\cline { 2 - 5 } & 0.0646 & 0.45 & 0.1790 & 0.25 \\
\hline Posterior & Expect. & Stand. & Expect. & Stand. \\
& Val. & Dev. & Val. & Dev. \\
& & & & \\
\hline & 0.2702 & 0.2046 & 0.2069 & 0.1639 \\
& & & & \\
\hline
\end{tabular}

Table 9. Composition of Posterior Distribution Project A

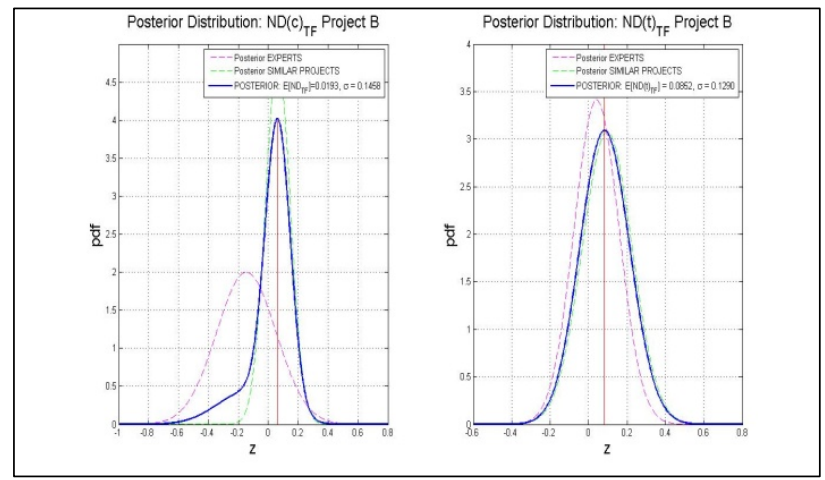

Figure 7. Posterior Distribution Project B

\begin{tabular}{|l|l|l|l|l|}
\hline \multicolumn{4}{|c|}{ Posterior Distribution Project B, $\varepsilon=0.2$} \\
\hline \multirow{2}{*}{} & \multicolumn{2}{|c|}{ ND(c) TF $^{|c|}$ ND(t) TF $^{\mid}$} \\
\hline Prior & Expect. & Stand. & Expect. & Stand. \\
& Val. & Dev. & Value & Dev. \\
\cline { 2 - 5 } & 0.0476 & 0.1373 & 0.0970 & 0.1390 \\
\hline
\end{tabular}




\begin{tabular}{|l|l|l|l|l|}
\hline Likelihood & $N D_{T N}$ & $\sigma_{T N}$ & $N D(t)_{T N}$ & $\sigma_{T N}$ \\
\cline { 2 - 5 } & -0.5956 & 0.55 & 0.0101 & 0.35 \\
\hline Posterior & Expect. & Stand. & Expect. & Stand. \\
& Val. & Dev. & Value & Dev. \\
\hline & 0.0193 & 0.1458 & 0.0852 & 0.1290 \\
\hline
\end{tabular}

Table 10. Composition of Posterior Distribution Project B

The posterior distribution of $\mathrm{ND}(\mathrm{c})_{\mathrm{TF}}$ is still strongly skewed to the left. The cost underrun recorded at time now tends to diminish the expected value of the posterior distribution in comparison with the prior one. On the other hand, the posterior distribution of $\mathrm{ND}(\mathrm{t})_{\mathrm{TF}}$ is quite symmetrical compared to the mode. Even in this case the expected value diminishes from the prior to the posterior because of the time performance observation at time now.

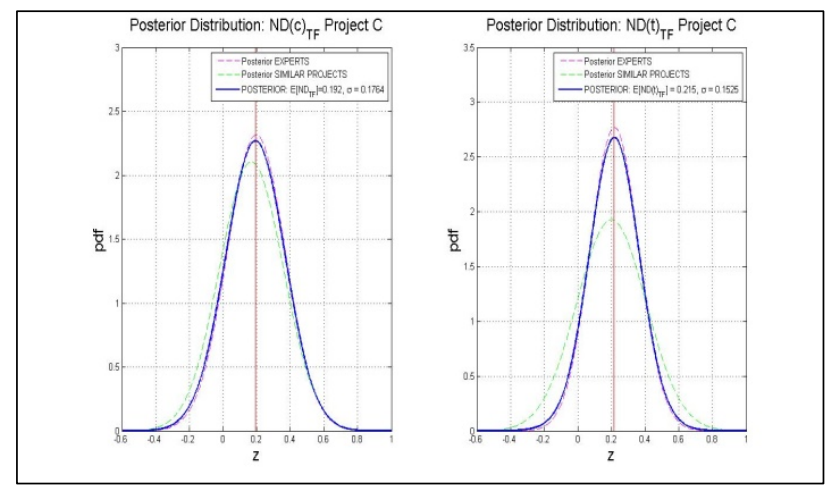

Figure 8. Posterior Distribution Project C

\begin{tabular}{|l|l|l|}
\hline \multicolumn{3}{|c|}{ Posterior Distribution Project $C, \varepsilon=0.8$} \\
\hline & $\mathrm{ND}(\mathrm{c})_{\mathrm{TF}}$ & $\mathrm{ND}(\mathrm{t})_{\mathrm{TF}}$ \\
\hline
\end{tabular}




\begin{tabular}{|l|c|c|c|c|}
\hline Prior & $\begin{array}{c}\text { Expect. } \\
\text { Val. }\end{array}$ & Stand. & Expect. & Stand. \\
& & & Val. & Dev. \\
\cline { 2 - 5 } & 0.2501 & 0.2040 & 0.2646 & 0.1828 \\
\hline Likelihood & $N D_{T N}$ & $\sigma_{T N}$ & $N D(t)_{T N}$ & $\sigma_{T N}$ \\
\cline { 2 - 5 } & 0.0199 & 0.35 & 0.0208 & 0.35 \\
\hline Posterior & Expect. & Stand. & Expect. & Stand. \\
& Val. & Dev. & Val. & Dev. \\
\cline { 2 - 5 } & & & & \\
\cline { 2 - 5 } & 0.1920 & 0.1764 & 0.2150 & 0.1525 \\
& & & & \\
\hline
\end{tabular}

Table 11. Composition of Posterior Distribution Project C

From Tab.11 it can be noticed that in both cases, i.e. cost and time, the standard deviation and the expected value of the posterior distribution are lower than those in the prior distribution. The expected value is lowered because of the observations at time now and the standard deviation because of the increasing confidence about the posterior expected value.

\subsection{Accuracy of the Bayesian model}

After projects $A, B$ and $C$ reached completion it was possible to check the error made by the Bayesian model and to compare it with the one that would have been made if traditional EVM forecasting formulas had been applied. Table 12 shows the mean squared error made by the Bayesian Model and by EVM in the three Oil and Gas cases.

\begin{tabular}{|l|c|c|}
\hline Mean & Bayesian & \\
Squared & Model & EVM \\
Error & & \\
\hline
\end{tabular}




\begin{tabular}{|l|c|c|}
\hline MSE Cost & $1.23 \cdot 10^{-2}$ & 0.1683 \\
\hline MSE Time & $9.3 \cdot 10^{-3}$ & $3.55 \cdot 10^{-2}$ \\
& & \\
\hline
\end{tabular}

Table 12. Comparison of the Mean Squared Error

It is clear that the Bayesian model is more accurate: its mean squared error is 10 times lower, both for cost and time performance, due to the larger amount of information used, even though the model was tested on very complex and risky projects.

\section{Conclusions}

In this paper a Bayesian model has been developed to support cost and schedule estimate at completion for a project. The main purposes are:

- to increase the forecasting accuracy with respect to the traditional EVM methodology, mainly for projects which are denoted by a high degree of uncertainty and complexity;

- to obtain more reliable forecasts in particular at the early phase of project execution in order to reduce any planning bias.

Firstly, the paper focuses on the need of integrating different information sources in the forecasting process, in particular the opinions of a group of experts and the statistics from the cluster of projects similar to the ongoing one. Experts' opinions allow for a forward looking analysis of the project development. To elicit the experts' opinions a procedure based on Analytic Hierarchy Process has been adopted. This methodology guarantees a high level of robustness in the elicitation process, minimizing, at the same time, the possible inconsistence of judgments. On the other side, the cluster of similar projects provides an "outside" point of view and mitigates any over-optimism bias. The selection of similar projects has to be carried out carefully based on the similarity criteria defined by the decision-maker. Lastly, the project performance values measured at time now, correspond to the traditional input to the forecasting process.

The Bayesian model developed in the paper allows for the rigorous integration of the different information sources. In particular, experts' opinions and similar past projects contribute to the prior 
distribution while the performance values measured at time now are the input for the likelihood function.

The Bayesian model has been applied to develop the estimate at completion of three Oil and Gas projects, featuring a high level of uncertainty and complexity. These projects represent a good challenge for the model. Comparing the accuracy of the Bayesian model with the one based on the traditional EVM, it has been observed that a drastic reduction of the mean squared error has resulted, even in the case of the project with the lowest progress at Time Now.

The model is being translated into a software application allowing for an easier use by the project managers.

Finally, it is important to highlight two main aspects. The model gives a high level of flexibility which is exemplified by the variety of possible shapes of the prior distribution that could assume even a bimodal shape. In the second instance, the easy evaluation of the expected value and of the standard deviation of the posterior distribution, without any need to utilize complex simulations.

Future development could mainly concern a further validation of the Bayesian model in other industries, evaluating the accuracy of the Bayesian model on a wider set of projects and testing different similarity criteria.

\section{Appendix}

The extensive expression of the posterior distribution can be found in Eq. (a.1) and Eq. (a.2):

$$
\begin{gathered}
\Pi(\mu \mid \mathrm{x})=\frac{\Pi(\mu) \cdot \mathrm{L}_{\mathrm{x}}(\mu)}{\int\left[\Pi(\mu) \cdot \mathrm{L}_{\mathrm{x}}(\mu)\right] \mathrm{d} \mu} \\
\Pi(\mu \mid \mathrm{x})=\frac{(1-\varepsilon) \cdot \Pi_{S}(\mu) \cdot \mathrm{L}_{\mathrm{X}}(\mu)+\varepsilon \cdot \Pi_{E}(\mu) \cdot \mathrm{L}_{\mathrm{x}}(\mu)}{\int\left[(1-\varepsilon) \cdot \Pi_{S}(\mu) \cdot \mathrm{L}_{\mathrm{X}}(\mu)+\varepsilon \cdot \Pi_{\mathrm{E}}(\mu) \cdot \mathrm{L}_{\mathrm{X}}(\mu)\right] \mathrm{d} \mu}
\end{gathered}
$$


In our specific case, Eq. (a. 2) can be simplified considering the posterior distribution typically obtained when the prior distribution and the likelihood are both normally distributed and the likelihood has known variance 0 .

For this purpose, the posterior distribution of similar projects is defined as in Eq. (a. 3):

$$
\begin{array}{r}
\Pi_{\mathrm{S}}(\mu \mid \mathrm{x})=\frac{\Pi_{\mathrm{S}}(\mu) \cdot \mathrm{L}_{\mathrm{x}}(\mu)}{\int\left[\Pi_{\mathrm{S}}(\mu) \cdot \mathrm{L}_{\mathrm{x}}(\mu)\right] \mathrm{d} \mu} \\
\Pi_{\mathrm{S}}(\mu \mid \mathrm{x}): \mathrm{N}\left(\frac{\sigma_{\mathrm{TN}}{ }^{2} \cdot \mu_{\mathrm{S}}+\sigma_{\mathrm{S}}{ }^{2} \cdot \mathrm{x}}{\sigma_{\mathrm{TN}}{ }^{2}+\sigma_{\mathrm{S}}{ }^{2}}, \frac{{\sigma_{\mathrm{TN}}}^{2} \cdot \sigma_{\mathrm{S}}{ }^{2}}{\sigma_{\mathrm{TN}}{ }^{2}+\sigma_{\mathrm{S}}{ }^{2}}\right)
\end{array}
$$

As appears from Eq. (a. 4), the parameters of the distribution $\Pi_{S}(\mu \mid x)$ can be obtained after:

- defining the parameters of the prior distribution of similar projects $\Pi_{S}(\mu)$;

- monitoring the time or cost performance of the ongoing project at the time now: $\mathrm{ND}_{\mathrm{TN}}$;

- $\quad$ assigning a standard deviation to the likelihood function $\sigma_{\mathrm{TN}}$, according to Tab. 3.

Analogously $\Pi_{E}(\mu \mid x)$ can be defined, i.e. the posterior distribution of experts' opinions. Its parameters of mean and variance are shown in Eq. (a. 5):

$$
\Pi_{\mathrm{E}}(\mu \mid \mathrm{x}): \mathrm{N}\left(\frac{\sigma_{\mathrm{TN}}^{2} \cdot \mu_{\mathrm{S}}+\sigma_{\mathrm{E}}^{2} \cdot \mathrm{x}}{\sigma_{\mathrm{TN}}{ }^{2}+\sigma_{\mathrm{E}}{ }^{2}}, \frac{\sigma_{\mathrm{TN}}^{2} \cdot \sigma_{\mathrm{E}}{ }^{2}}{\sigma_{\mathrm{TN}}{ }^{2}+\sigma_{\mathrm{E}}{ }^{2}}\right)
$$

In order to obtain a further simplification of Eq. (a. 2), the two terms $D_{E}$ e $D_{S}$ are defined in the following:

$$
\begin{aligned}
& D_{E}=\int\left[\Pi_{E}(\mu) \cdot L_{x}(\mu)\right] d \mu \\
& D_{S}=\int\left[\Pi_{S}(\mu) \cdot L_{x}(\mu)\right] d \mu
\end{aligned}
$$

Developing Eq. (a. 6) and Eq. (a.7) the analytical expressions for $\mathrm{D}_{\mathrm{E}}$ and $\mathrm{D}_{\mathrm{S}}$ are obtained: 


$$
\begin{aligned}
& D_{E}=\frac{1}{\sqrt{2 \pi\left(\sigma_{\mathrm{E}}^{2}+\sigma_{\mathrm{TN}}^{2}\right)}} \cdot e^{-\frac{1}{2}\left(\frac{\mathrm{x}-\mu_{\mathrm{E}}}{\sqrt{\left(\sigma_{\mathrm{E}}^{2}+\sigma_{\mathrm{TN}}^{2}\right)}}\right)^{2}} \\
& D_{\mathrm{S}}=\frac{1}{\sqrt{2 \pi\left(\sigma_{\mathrm{S}}^{2}+\sigma_{\mathrm{TN}}^{2}\right)}} \cdot \mathrm{e}^{-\frac{1}{2}\left(\frac{\mathrm{x}-\mu_{\mathrm{S}}}{\sqrt{\left(\sigma_{\mathrm{S}}^{2}+\sigma_{\mathrm{TN}}{ }^{2}\right)}}\right)^{2}}
\end{aligned}
$$

Thus Eq. (a. 8) and (a. 9) can be introduced in Eq. (a.1) as follows.

$$
\Pi(\mu \mid x)=\frac{(1-\varepsilon) \cdot\left[\Pi_{S}(\mu) \cdot L_{X}(\mu)\right]+\varepsilon \cdot\left[\Pi_{E}(\mu) \cdot L_{X}(\mu)\right]}{(1-\varepsilon) \cdot D_{S}+\varepsilon \cdot D_{E}}
$$

Let us multiply the first and the second member of the numerator respectively times $\frac{D_{S}}{D_{S}}$ and $\frac{D_{E}}{D_{E}}$; by doing so, $\Pi_{S}(\mu \mid x)$ e $\Pi_{E}(\mu \mid x)$ can be identified in Eq. (a. 11), obtaining Eq. (a. 12):

$$
\begin{array}{r}
\Pi(\mu \mid x)=\frac{(1-\varepsilon) \cdot\left(\frac{\Pi_{S}(\mu) \cdot L_{X}(\mu)}{D_{S}}\right) \cdot D_{S}+\varepsilon \cdot\left(\frac{\Pi_{E}(\mu) \cdot L_{X}(\mu)}{D_{E}}\right) \cdot D_{E}}{(1-\varepsilon) \cdot D_{S}+\varepsilon \cdot D_{E}} \\
\Pi(\mu \mid X)=\frac{(1-\varepsilon) \cdot \Pi_{S}(\mu \mid X) \cdot D_{S}+\varepsilon \cdot \Pi_{E}(\mu \mid X) \cdot D_{E}}{(1-\varepsilon) \cdot D_{S}+\varepsilon \cdot D_{E}}
\end{array}
$$

Evidencing $\Pi_{S}(\mu \mid x)$ and $\Pi_{E}(\mu \mid x)$ in Eq. (a. 12), $\Pi(\mu \mid x)$ can be evaluated as the sum of two contributions:

$$
\Pi(\mu \mid \mathrm{x})=\frac{(1-\varepsilon) \cdot \mathrm{D}_{\mathrm{S}}}{(1-\varepsilon) \cdot \mathrm{D}_{\mathrm{S}}+\varepsilon \cdot \mathrm{D}_{\mathrm{E}}} \cdot \Pi_{\mathrm{S}}(\mu \mid \mathrm{x})+\frac{\varepsilon \cdot \mathrm{D}_{\mathrm{E}}}{(1-\varepsilon) \cdot \mathrm{D}_{\mathrm{S}}+\varepsilon \cdot \mathrm{D}_{\mathrm{E}}} \Pi_{\mathrm{E}}(\mu \mid \mathrm{x})
$$

From Eq. (a. 13), it can be noticed that a "posterior weight" can be defined: it operates a mixture between the posterior distribution of similar projects and the posterior distribution of experts' opinions.

The posterior weight has the following expression: 


$$
\varepsilon^{*}=\frac{\varepsilon \cdot D_{\mathrm{E}}}{(1-\varepsilon) \cdot \mathrm{D}_{\mathrm{S}}+\varepsilon \cdot \mathrm{D}_{\mathrm{E}}}
$$

Analyzing Eq. (a. 14) and recalling Eq. (a. 8) and Eq. (a. 9) of $\mathrm{D}_{\mathrm{E}}$ and $\mathrm{D}_{\mathrm{S}}$, it can be observed that the posterior weight $\varepsilon^{*}$ depends from the initial weight $\varepsilon$, the performance of the ongoing project observed at time now $\mathrm{x}$, the standard deviation $\sigma_{\mathrm{TN}}$ of the likelihood function, and from the parameters of the prior distribution of experts' opinions and similar projects:

$$
\varepsilon^{*}=\mathrm{f}\left(\varepsilon, \mathrm{x}, \sigma_{\mathrm{TN}}, \mu_{\mathrm{E}}, \sigma_{\mathrm{E}}, \mu_{\mathrm{s}}, \sigma_{\mathrm{s}}\right)
$$

It can be noticed that the multiplicative term of $\Pi_{S}(\mu \mid x)$ in Eq. (a.13) equals $1-\varepsilon^{*}$. Let us demonstrate it, showing the detailed calculations:

$$
\begin{gathered}
1-\varepsilon^{*}=1-\frac{\varepsilon \cdot D_{E}}{(1-\varepsilon) \cdot D_{S}+\varepsilon \cdot D_{E}}=\frac{\left[(1-\varepsilon) \cdot D_{S}+\varepsilon \cdot D_{E}\right]-\varepsilon \cdot D_{E}}{(1-\varepsilon) \cdot D_{S}+\varepsilon \cdot D_{E}}= \\
1-\varepsilon^{*}=\frac{(1-\varepsilon) \cdot D_{S}}{(1-\varepsilon) \cdot D_{S}+\varepsilon \cdot D_{E}}
\end{gathered}
$$

Therefore, the final formula of the posterior distribution in Eq. (a. 2), after the proper elaborations, is expressed in Eq. (a. 17):

$$
\left.\Pi(\mu \mid \mathrm{x})=\left(1-\varepsilon^{*}\right) \cdot \Pi_{\mathrm{S}}(\mu \mid \mathrm{x})+\varepsilon^{*} \cdot \Pi_{\mathrm{E}}(\mu \mid \mathrm{x}) \quad \text { (a. } 17\right)
$$

Hence the posterior distribution results to be a new mixture of the two informative sources, evaluated $a$ posteriori, through a posterior weight " $\varepsilon^{* ”}$.

Starting from the Eq. (a. 17) the expected value and the standard deviation of the posterior distribution can be easily evaluated by applying the statistical definition of these two quantities. 


\section{References}

Anbari, F., (2003) Earned Value: Project management method and extension, Project Management Journal 34, 12-23.

Brandon, D.M. (1998) Implementing Earned Value easily and effectively, Project Management Journal 29, 11-18.

Caron, F., F. Ruggeri and A. Merli (2013) A Bayesian approach to improve estimate at completion in Earned Value Management, Project Management Journal (44), 3-16.

Caron, F. (2014) Managing the continuum: certainty, uncertainty, unpredictability in large engineering projects, Springer

Christensen, D. (1996), Project Advocacy and the estimate at completion problem, Journal of Cost Analysis and Management, Spring 1996, 35-60

Daneshkhah, A.R., Psychological Aspects Influencing Elicitation of Subjective Probability, Research Report, University of Sheffield, 2004.

De Finetti, B. (1937) La prévison: ses lois logiques, ses sources subjectives, Annales de l'Institut Henri Poincaré, 7,1 1-68.

Dvir, D. and Lechler, T. (2004), Plans are nothing, changing plans is everything: the impact of changes on project success, Research Policy, 33, 1-15

El-Sabban, M.Z. (1973), Forecast of cost/schedule status utilizing cost performance reports of the Cost/Schedule Control Systems Criteria: a Bayesian approach, US Army Aviation Systems Command, St. Louis, Missouri, (AD-754576).

Fleming, Q.W., J. Koppelman (2006) Earned value project management $3^{\text {rd }}$ ed., Project Management Institute, Newton Square, PA,

Flyvberg, B. (2006) From Nobel Prize to project management: Getting risk right. Project Management Journal, 37, 5-15. 
Flyvbjerg, B. (2009), Survival of the un-fittest: why the worst infrastructure gets built - and what we can do about it, Oxford Review of Economic Policy, 25, 3, 344-367.

Forman, E., K. Peniwati, (1998) Aggregating individual judgments and priorities with the Analytic Hierarchy Process, European Journal of Operational Research,108), 165-169.

Gardoni, P., Reinschmidt K.F. and R. Kumar (2007) A probabilistic framework for Bayesian adaptive forecast, Computer-Aided Civil and Infrastructure Engineering, 22, 182-196.

Goodwin, P. (2005) How to integrate management judgment with statistical forecasts, Foresight, 1, 8-12

Green, K.C. and Armstrong, J.S. (2007) Structured analogies for forecasting, International Journal of Forecasting, 23, 365-367

Haji-Kazemi, S., Andersen, B. and Krane, H.P. (2013), A review on possible approaches for detecting early warning signs in projects, Project management journal, 44, 5, 55-69.

Henderson, K. Further developments in Earned Schedule, The Measurable News (Spring 2004), 15-20.

Hogarth, R.M. and Makridakis, S. (1981) Forecasting and planning: an evaluation, Management Science, 27, 2, 115-138

Kahneman, D. and Tversky, A. (1979) Intuitive prediction: biases and corrective procedures, TIMS Studies in Management Science, 12, 313-327.

Kim, B., K.F. Reinschmidt (2009), Probabilistic forecasting of project duration using Bayesian inference and the beta distribution, Journal of Construction Engineering and Management 135, 3, 178-186.

Kim, B. (2015) Integrating risk assessment and actual performance for probabilistic project cost forecasting: a second moment Bayesian model, IEEE Transactions on Engineering Management, $62,2,158-170$

Kuosa, T. (2012) The evolution of strategic foresight, Gower

Lipke, W. (2002a). A study of the normality of Earned Value Management indicators. The Measurable News, December 2002, 1-16, 
Lipke, W. (2002b). Statistical process control of project performance, Crosstalk-The Journal of Defense Software Engineering, 13, March 2002, 16-20,

Lipke, W. (2003). Schedule is different, The Measurable News, Summer 2003, 31-34,

Lipke, W. (2006a). Earned Schedule Leads to Improved Forecasting, Proceedings of the 3rd international conference on project management (PROMAC 2006), September 2006

Lipke, W. (2006b). Statistical methods applied to EVM ...the next frontier. The Measurable News, Winter 2006, 18-30.

Liu, L. and Zhu, K. (2007) Improving cost estimates of construction projects using phased cost factors, Journal of construction engineering and management, 133, 1, January 1

Lovallo, D. and Kahneman, D. (2003), Delusion of Success: How optimism undermines executives' decisions, Harvard Business Review, 81, 56-63.

Lovallo, D., Clarke C. and Camerer C. (2012) Robust analogizing and the outside view: two empirical tests of case-based decision making. Strategic Management Journal, 33, 496-512

Maylor, H., Project Management 4th ed., Pearson Education, Harlow, 2010.

Marshall, R.A., Ruiz, P. \& Bredillet, C.N. (2008). Earned value management insights using inferential statistics, International Journal of Managing Projects in Business,1(2), 288-294

Merrow, E.W. (2011) Industrial Megaprojects: Concepts, strategies and practices for success, John Wiley \& Sons, Hoboken, New Jersey.

O'Hagan, A., C. Buck, (2006) Uncertain Judgments: eliciting expert probabilities, John Wiley \& Sons, Chichester

Palomo, J., Ruggeri, F., Rios Insua, D., Cagno, E., Caron, F. and Mancini, M. (2006) On Bayesian forecasting of procurement delays: a case study, Applied Stochastic Models in Business and Industry, 22, 181-192

Project Management Institute, (2013) A guide to the Project Management Body of Knowledge (PMBOK) 5th ed., Project Management Institute, Newton Square, PA

Rose, K.H. (2003) Review of earned value project management, Project Management Journal Reich, B.H., Gemino, A. and Sauer, C. (2014) How knowledge management impacts performance in projects: an empirical study, International Journal of Project Management, 32,4, 590-602 
Savio, N.D. and Nikoloupolos K. (2011) A strategic forecasting framework for governmental decision making and planning, International Journal of Forecasting, available on line November 2011

Schindler, M. and Eppler, M.J. (2003) Harvesting project knowledge: a review of project learning methods and success factors, International Journal of Project Management, 21, 219-228 Saaty, L. T. (2011) Fundamentals of decision making and priority theory with the Analytic Hierarchy Process-Vol. VI of the AHP series, RWS Publications, Pittsburgh.

Vanhoucke, M., S. Vandevoorde, (2006), A comparison of different project duration forecasting methods using earned value metrics, International Journal of Project Management, 24 pp. 289-302. Williams, T., Klakegg, O.J., Walker, D. H.T., Andersen, B. and Magnussen, O.M. (2012), Identifying and Acting on Early Warning Signs in Complex Projects, Project Management Journal, 43, 2, 3753 